Article

\title{
Jumping Side Volley in Soccer-A Biomechanical Preliminary Study on the Flying Kick and Its Coaching Know-How for Practitioners
}

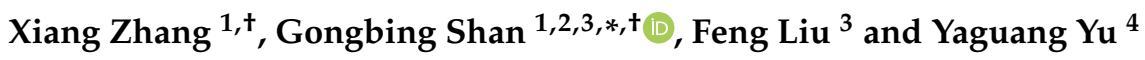 \\ 1 Department of Physical Education, Xinzhou Teachers' University, Xinzhou 034000, China; \\ xiangzhang@xztc.edu.cn \\ 2 Biomechanics Lab, Faculty of Arts \& Science, University of Lethbridge, Lethbridge, AB T1K 3M4, Canada \\ 3 School of Physical Education, Shaanxi Normal University, Xi'an 710119, China; liufeng668@snnu.edu.cn \\ 4 College of Martial Arts, Shandong Sport University, Rizhao 276800, China; yuyaguang@sdpei.edu.cn \\ * Correspondence: g.shan@uleth.ca; Tel.: +1-403-329-2683 \\ + The authors contributed equally to this work.
}

Received: 4 May 2020; Accepted: 10 July 2020; Published: 12 July 2020

\begin{abstract}
The jumping side volley has created breathtaking moments and cherished memories for soccer fans. Regrettably, scientific studies on the skill cannot be found in the literature. Relying on the talent of athletes to improvise on the fly can hardly be considered a viable learning method. This study targeted to fill this gap by quantifying the factors of the jumping side volley and to contribute to the development of a coaching method for it. Using 3D motion capture (12 cameras, $200 \mathrm{~Hz})$ and full-body biomechanical modeling, our study aimed to identify elements that govern the entrainment of skill execution. Given the rarity of players who have acquired this skill and the low success rate of the kick (even in professional games), we were able to achieve and review 23 successful trials from five college-level subjects and quantify them for the study. The results unveiled the following key elements: (1) the control of trunk rotation during jumping, (2) the angle between thighs upon take-off, (3) the whip-like control of the kicking leg while airborne, (4) timing between ball motion and limb coordination, and (5) damping mechanism during falling. An accurate kick can normally be achieved through repetitive training. This underlines the need for athletes to master a safe landing technique that minimizes risk of injury during practice. Therefore, training should begin with learning a safe falling technique.
\end{abstract}

Keywords: 3D motion capture; full-body biomechanical modeling; X-factor; hip flexibility; whip-like movement; dispersion of impact load during falling

\section{Introduction}

The great attraction of soccer for millions of fans may trace back to the basic idea of the game: the goal-an idea that never ceases to fascinate. Compared to many other sports, goals are relatively rare in soccer (on average $<3$ goals/game in the FIFA World Cup since the 1960s [1]). Because of the rarity, soccer goals are extremely exciting. The game can be thought of as an improvised drama, where emotional tension is built over long periods only to be fully released when a goal is achieved. In particular, the goals achieved by applying flying techniques such as the diving scorpion kick, bicycle kick, diving header, and jumping side volley are sources of rabid excitement. This uniqueness of soccer contributes to making the game the number one sport worldwide [2-5]. Among all the techniques, the jumping side volley is, no doubt, one of the infrequent scoring skills that fans invariably desire to see when attending games. Unfortunately, few players have performed this skill during national or international tournaments. 
The jumping side volley is an acrobatic airborne technique (Figure 1). One can see its rarity from the European Championship 2016 (Euro 2016) where it was used for only one out of 108 goals [6]. However, this rare skill has created breathtaking moments and cherished memories for players and fans. A classic example is Wendell Lira's (Brazil) superb airborne side volley, which won the most prestigious FIFA Puskás Award 2015 [7]. In Euro 2016, Xherdan Shaqiri's (Swiss) jumping side volley was selected as the best goal of the tournament [8]. The novelty of the skill (just like the acrobatic bicycle kick) and its rarity are because these kicks are perceived as high-risk and low-return skills [9]. Regrettably, a scientific study on the skill cannot be found in the literature [4]. Relying on the talent of athletes to improvise on the fly can hardly be considered as a viable learning strategy. Therefore, in order to give efficient and effective information to practitioners, we launched a preliminary study [10] to give a scientific overview of the kick, to identify key features of the skill, and to formulate a scientific way of learning/training the technique. Specifically, this preliminary study had two objectives: (1) use of 3D motion capture technology to quantify the dominant factors contributing to the kick quality and (2) identify biomechanical elements that govern the entrainment of the jumping side volley in order to develop its coaching method.
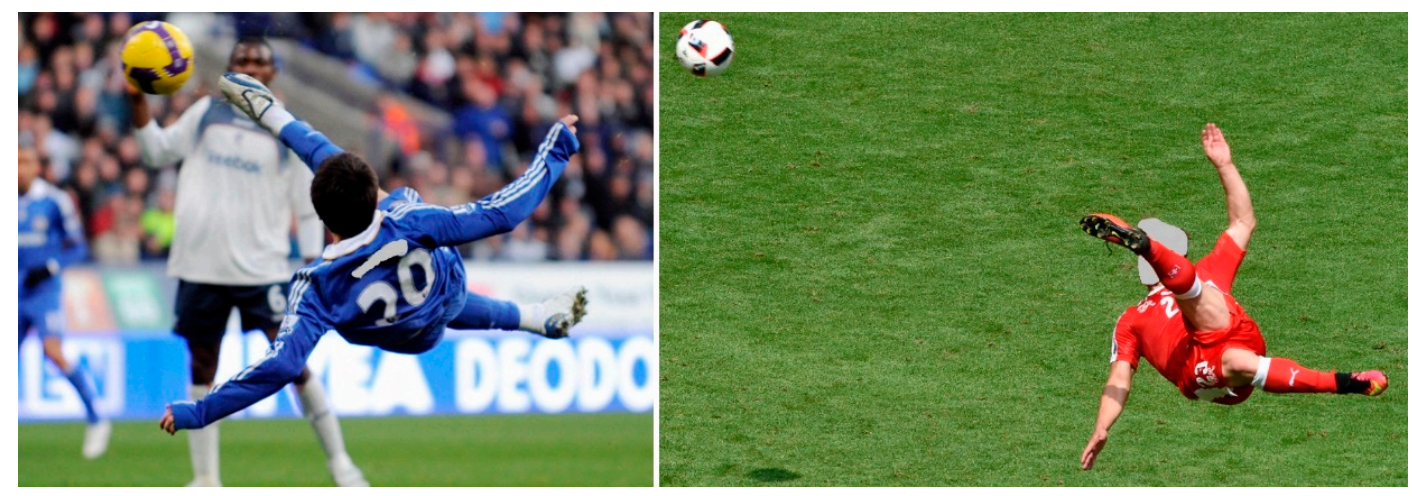

Figure 1. Jumping side volley—a flying techniques for defeating goalies.

\section{Materials and Methods}

\subsection{How to Establish a Lab Test Condition for Mimicking Reality in an Application-Oriented Investigation?}

Since the study was an application-oriented investigation, the first challenge was how to gather realistic data. We went back to professional games to find a reasonable solution.

In reality, ball flying-in direction is arbitrary. Figure 2 supplies four successful examples selected from professional soccer games. Example 1 (Figure 2a) shows that the player used his head to set a flying-in ball vertically and then performed a jumping side volley. In examples 2 and 3 (Figure 2b,c), the player employed his chest and foot, respectively, to set a flying-in ball vertically for doing a jumping side volley. A vertically travelling ball can also be set by other players (Figure $2 \mathrm{~d}$, the goal keeper set a vertically travelling ball for a player). All four examples demonstrate that the vertically travelling ball is a frequent scenario for executing the jumping side volley. Therefore, the vertically travelling ball was used for each subject in our lab-based data collection. 


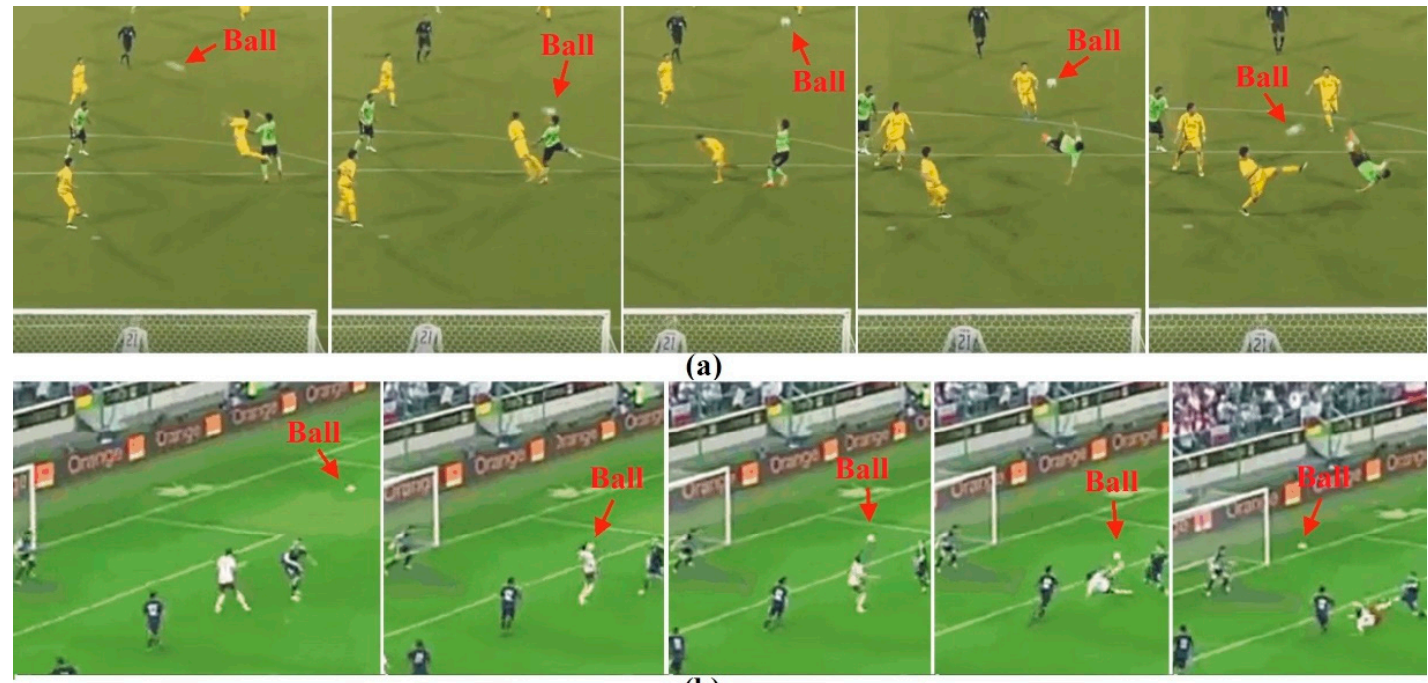

(b)

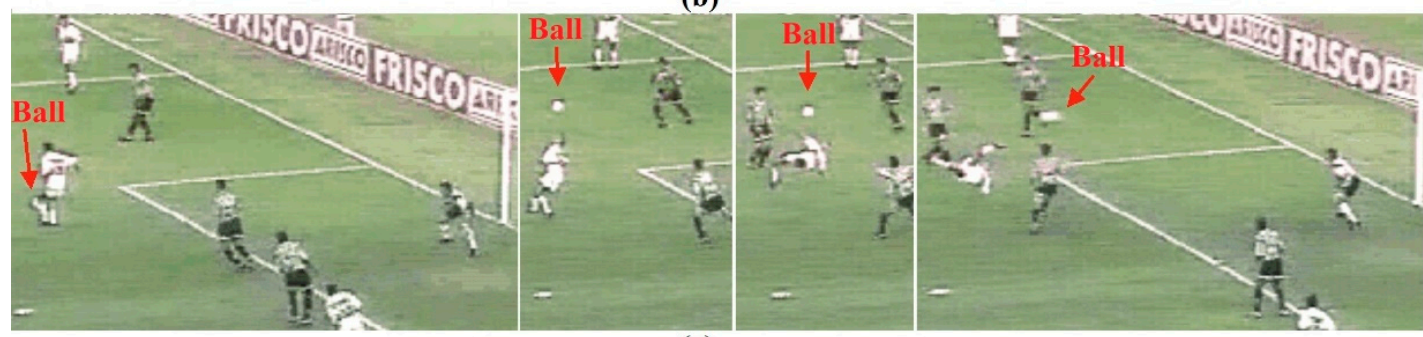

(c)

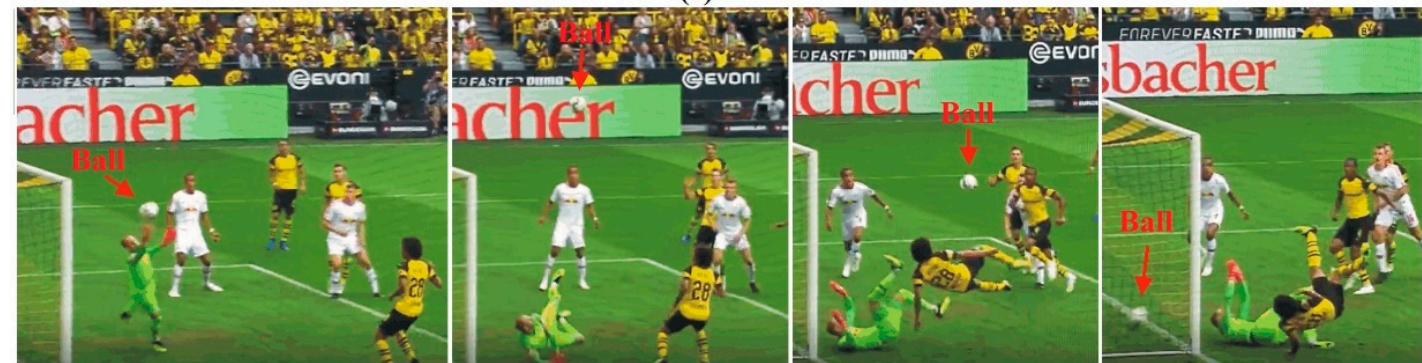

(d)

Figure 2. Successful examples of the jumping side volley in professional games. The common feature of the examples is a vertically travelling ball. The ball condition can be created by a player using (a) his head; (b) his chest; (c) his foot; or (d) set by another player.

\subsection{Motion Capture and Biomechanical Modeling}

A 3D, twelve-camera VICON MX40 motion-capture system (VICON Motion Systems, Oxford Metrics Ltd., Oxford, England [11]) was used to measure the jumping side volley using 42 reflective, $9 \mathrm{~mm}$ markers on the body. The motion capture system tracked the markers at a rate of 200 frames/s. This capture rate has been widely applied in various analyses of complicated/elite sport-skills [12-16]. Figure 3 shows a 3D computer reconstruction of a single trial, including camera placement, capture volume, and a rendered stick figure. Markers were placed on subjects as follows: (1) four on the head, (2) trunk markers on the sternal end of the clavicle, xiphoid process of the sternum, C7 and T10 vertebrae, each scapula, left and right anterior superior iliac spine, posterior superior iliac spine, (3) upper-extremity markers on the right and left acromion, lateral side of upper arm, lateral epicondyle, lateral side of forearm, styloid processes of radius and ulna, and distal end of 3rd metacarpal bones, and (4) lower extremity markers on left and right lateral sides of thigh and shank, lateral tibial condyle, lateral malleolus, distal end of $5^{\text {th }}$ metatarsal, calcaneus, and big toe. Please note that markers on the scapula, upper arms, forearms, thighs, and shanks, are referential, therefore, no accurate positions were required, i.e., as long as they are over the required bones, they will work. For reducing the risk 
of injury, soft markers were applied for the tests. These compress (and decompress) easily, therefore, their influence on skill performance is negligible. Calibration residuals were determined in accordance with VICON's guidelines [11] and yielded positional data accurate within $1 \mathrm{~mm}$.

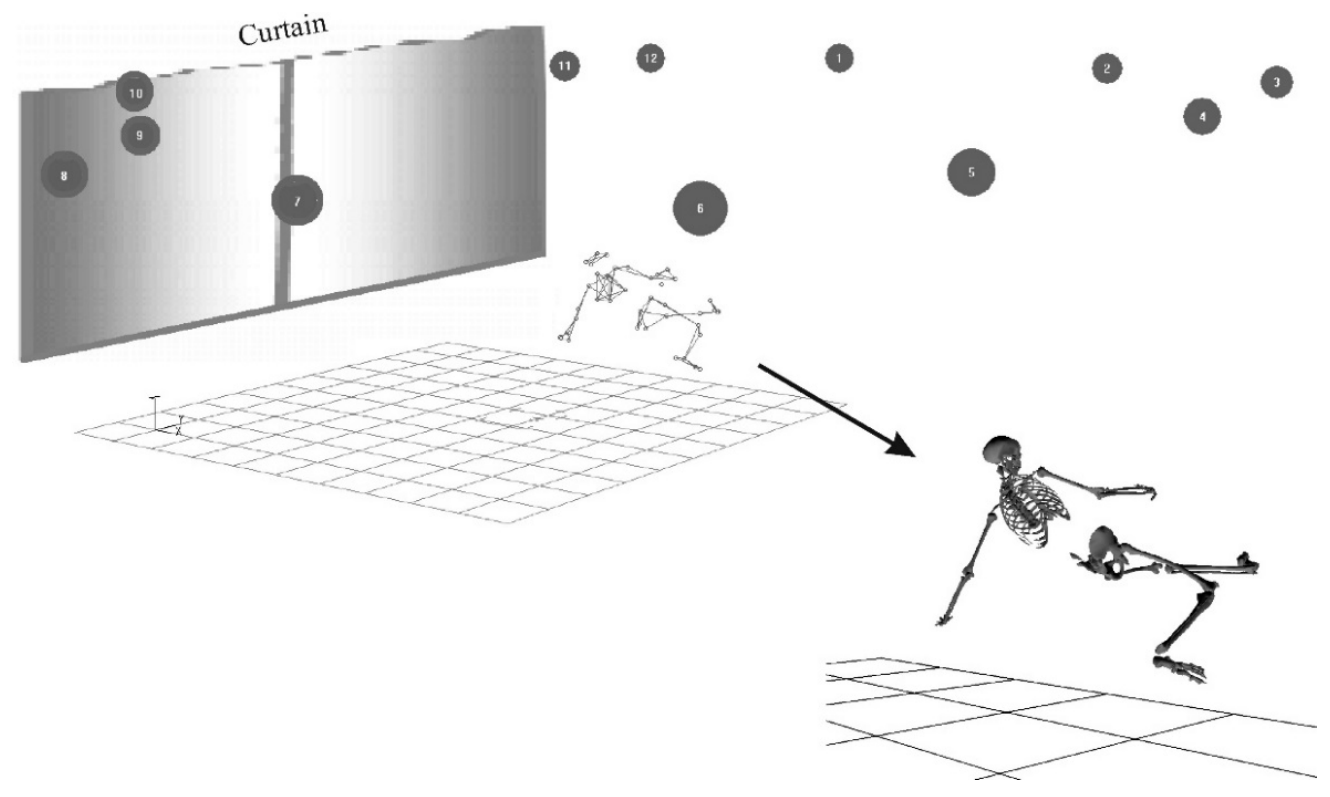

Figure 3. 3D motion capture reconstruction showing the 12 camera placements and a wire frame mesh reproduction of a jumping side volley (left) and a 15-segment biomechanical model built from the 3D data collected.

Additionally, three reflex markers (made from 3M reflective paper) were glued to the ball in order to quantify the soccer release speed.

VICON software triangulated the positions of each marker and rendered them in three-dimensional computer space. The raw data collected was processed by a five-point smoothing filter. The five-point filter was a premier filter in the time domain that reduced random noise while retaining a sharp step response [16]. It is widely applied to reduce noise from possible vibrations of the markers during 3D motion capture of sports and arts performances $[14,17,18]$. The resultant data supplied primary information, such as marker position, position changes, velocities, and accelerations. From the marker-position data, anatomical landmarks were established that allowed modeling of the skeletal structure for each participant. Using basic physics, simple positional data were translated into skeletal movement. VICON software provide tools for building a 15-segment biomechanical model of the soccer kick (Figure 3) [19-22]. Model segments were identified as follows: head, upper trunk, lower trunk, upper arms, lower arms, hands, thighs, shanks, and feet. The model calculated segment lengths, joint angles, and ranges of motion (ROMs) for the joints [23,24]. In such biomechanical modeling, inertial characteristics of the body are estimated using anthropometric norms found through statistical studies $[25,26]$. The modeling enables researchers to postulate motor control patterns. After model calculations, descriptive statistics (i.e., average and standard deviation) of body kinematic data (i.e., joint angles, joint ROMs, and coordination timing of joints) and correlation analyses among the body kinematic data with ball release speed were performed using EXCEL 2016. The ball release speed is commonly used to judge the kick quality [3-5], as such, the correlation analyses aimed to find the key/dominant factors among the kinematic data that could govern the entrainment of the jumping side volley.

Motion capture technology permits considerable freedom of movement for participants without negatively influencing their motor skill control. Taking advantage of this, we placed no restrictions on subjects' movements within the capture volume in an effort to preserve their normal "style". Given the 
rarity of individuals who have acquired this skill, we used the "self-identification" method in the search for subjects at three universities (all have the VICON system) and recruited 14 male soccer players. The subjects were informed of the testing procedures, signed consent forms, and voluntarily participated in the data collection. The universities' human-subject committees scrutinized and approved the test as meeting the criteria of ethical conduct for research involving human subjects. Through pretests, we found only five players who could actually perform this skill in the required test conditions, but not at a 100\% success-rate. Two of them were Canadian and the others were Chinese. The anthropometrical characteristics and experience in soccer training were as follows: body height $1.74 \pm 0.04 \mathrm{~m}$, body weight $70.4 \pm 3.8 \mathrm{~kg}$, age $22.0 \pm 1.6$ years, and training $15.8 \pm 1.5$ years. After warm-up, each subject was asked to perform the skill six times. In total, 23 successful trials (i.e., the ball was accurately and powerfully kicked) were captured.

\section{Results}

Figure 4 shows that two events-take-off and ball contact-divide the jumping side volley into three phases: (1) the jumping phase, (2) the airborne phase, and (3) the landing phase. Our data reveal the following characteristics of the 1st phase: Before the volley, the athlete's trunk and pelvis rotate away from the goal. During the takeoff, the non-kick-side (NKS) leg is raised, at the same time, the trunk reverses rotational directions and twists toward the goal. In order to increase the range of motion (ROM) of trunk rotation, both arms abduct to near horizontal (over $80^{\circ}$, Table 1). Until the take-off, the ROM of the trunk twist (commonly known as the X-factor [27-29]) is about $40^{\circ}$ (Table 1). At the end of the phase, the trunk-orientation approaches a more horizontal position (Figure 4). Correlation analyses confirm that the X-factor $(\alpha)$, angle between thighs $(\beta)$ at take-off, and shoulder abduction during jumping are key/dominant factors, influencing the kick quality, i.e., the ball release speed (Figure 5a,b).

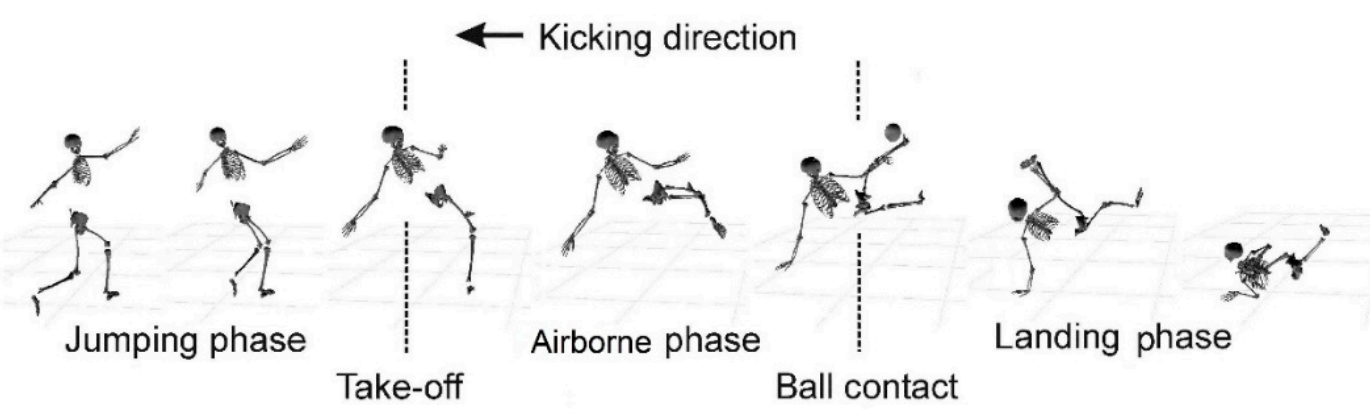

Figure 4. Phase identification based on 3D motion analysis data.

Table 1. Average and standard deviation of selected parameters, their confidence intervals (CI), coefficient of variation (CV), and their correlation with the ball release speed $(r)(p<0.05)$.

\begin{tabular}{|c|c|c|c|c|c|}
\hline \multicolumn{2}{|c|}{ Selected Parameters } & Results & CI & $\mathrm{CV}$ & $\mathbf{r}$ \\
\hline \multirow{3}{*}{$\operatorname{ROM}\left(^{\circ}\right)$} & Trunk Twist $(X$-factor $\alpha)$ & $41.5 \pm 5.1$ & $40.5-42.6$ & 0.12 & 0.86 \\
\hline & KS Hip (Flex/Ext) & $51.6 \pm 5.2$ & $50.5-52.7$ & 0.10 & 0.71 \\
\hline & KS Knee (Flex/Ext) & $56.9 \pm 6.1$ & $55.6-58.1$ & 0.11 & 0.76 \\
\hline \multirow{4}{*}{ Angle $\left(^{\circ}\right)$} & KS Ankle (Flex/Ext) & $11.6 \pm 2.2$ & $11.1-12.1$ & 0.19 & 0.52 \\
\hline & Max Shoulder Abduction * & $83.7 \pm 4.2$ & $82.8-84.5$ & 0.05 & 0.69 \\
\hline & $\begin{array}{c}\text { Take-off Angle between } \\
\text { Thighs }(\beta)\end{array}$ & $75.2 \pm 13.0$ & $72.5-77.9$ & 0.17 & 0.82 \\
\hline & Min KS Knee Angle & $84.1 \pm 9.6$ & $82.1-86.1$ & 0.11 & -0.75 \\
\hline Coordination & Trunk $\rightarrow$ KS Hip & $83.2 \pm 4.1$ & $82.3-84.1$ & 0.05 & 0.57 \\
\hline Timing (\%) & KS Hip $\rightarrow$ KS Knee & $74.4 \pm 15.4$ & $71.2-77.6$ & 0.21 & 0.89 \\
\hline
\end{tabular}

$X$-factor: the angle between shoulder line (upper trunk) and hip line (lower trunk). Flex/Ext: Flexion/Extension, KS: Kick Side. *: the max value is the average of both shoulders. 
Two notable characteristics of the 2nd phase (i.e., the airborne phase) are the flexed kick-side (KS) leg and the extended NKS leg (Figure 5c). Both legs form a scissor-movement, i.e., they move in opposite directions. The correlation analyses show that both the minimum angle and ROM of the KS knee are key factors affecting the kick quality (Table 1). From the timing perspective, the KS hip flexion starts when about $80 \%$ of the trunk twist toward the goal has finished. Similarly, the KS knee begins its extension after the KS hip finishes about $75 \%$ of its flexion (Table 1). Further, our data show that the explosive KS knee extension happens shortly before the ball contact, and is followed by an ankle flexion. The correlation analyses unveil that the sequential segment-coordination is also a key contributor to the kick quality.

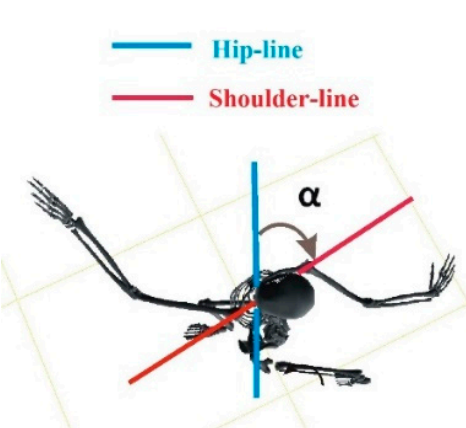

(a)

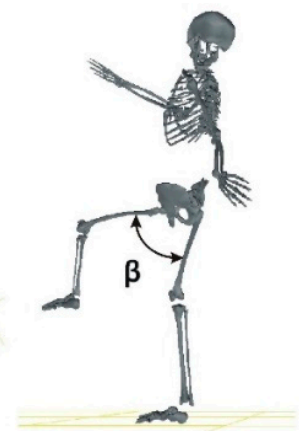

(b)

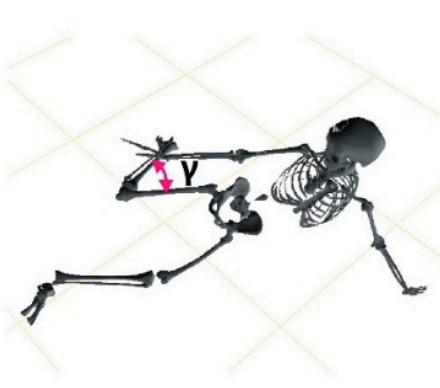

(c)

Figure 5. Key/dominate factors influencing kick quality: (a) X-factor $\alpha$ (top view); (b) Take-off angle between thighs $\beta$ (side view); (c) Minimum KS knee angle $\gamma$ (top-front view).

The main issue in the 3rd phase (i.e., the landing phase) is how to dissipate the impact load produced by falling in order to avoid potential injuries. Our data reveal that the well-trained athletes apply multiple landings to share the impact loads for reducing the risk of injury. The 1st landing is the flexed arm-hand chain (like a spring) for the 1st damping. The 2nd landing is the hip landing, followed by body rolling, sharing the rest load among multiple contact points.

\section{Discussion}

Since this study focuses on coaching practice, the results should be coach-friendly. A coach-friendly study, in our vision, should use a well-established scientific method to supply explanations on (1) scientifically-identified motor-control sequencing; (2) dominant factors (determined by correlation analyses) contributing to the control of the motor skill; and (3) instructions that can be understood by coaches and applied in their intervention in practice. Therefore, based on numerous analyses of our 3D data and their correlation analyses, the following key factors have been selected for illustrating the secrets of the control of the acrobatic skill in order to establish a practical way to learn and to train the jumping side volley:

- phase identification,

- trunk rotary control,

- hip flexibility,

- lower-limb control,

- upper-limb control, and

- coordination between upper- and lower-limbs.

A focused communication would help practitioners to understand the complex motor control in a timely and efficient way.

Regarding the determinants influencing the kick quality, the following key/dominant factors were revealed: There are two key factors in phase 1: the twisting control of the upper body $(\alpha)$ and the 
instance angle between the two thighs at take-off ( $\beta$ ). The larger $\alpha$ and $\beta$ are, the more powerful is the kick. Actually, these two factors play a crucial role in laying a foundation for performing the whip-like control of the kick in phase 2. In conjunction with the key factors in phase 2, the effects of $\alpha$ and $\beta$ are elaborated below.

One notable feature of phase 2 is the asymmetric control of the legs, i.e., multi-segment control of the kick leg vs. quasi-single-segment control of the non-kick leg. This asymmetric control results in a difference between the moments of inertia of the two legs. The difference is vital for forwardly accelerating the kick leg; as such, it influences the quality of the whip-like movement. In the airborne phase, the human body follows the Law of Conservation of Angular Momentum. That means, the angular momentum of the forward action (the KS leg) equals the angular momentum of the backward reaction (the NKS leg). The flexed KS leg leads to a smaller moment of inertia (I), resulting in a faster forward motion in comparison to the extended NKS leg (a larger I creates lower backward motion) (Figure 6). In conjunction with phase 1, we found that the whip-system of the jumping side volley consists of four segments: trunk, KS-thigh, KS-shank, and kick foot. The whip-like kick is actually initiated in phase 1 , beginning with the trunk twisting toward the goal, followed by hip flexion, knee extension, and ankle flexion, showing a sequential flow of energy and momentum transfer. It is well known that increasing the ROM of each segment will enhance the effect of the whip-like movement [14,19,28-31]. Therefore, flexibility of hip and knees should be emphasized during training, and the training should also pay attention to segment coordination (i.e., the whip-like control). Of course, timing is the most crucial element for coordination training.

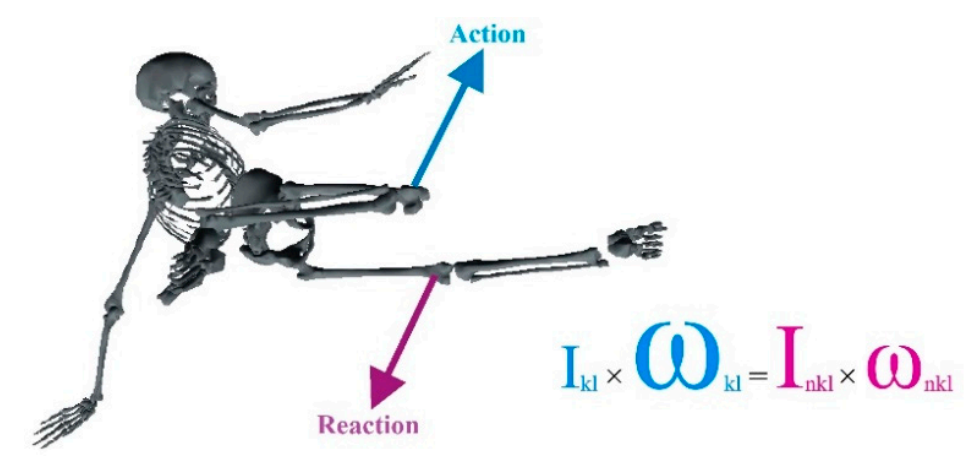

Figure 6. The asymmetric control of the legs during the airborne phase and their effects revealed by the Law of Conservation of Angular Momentum, i.e., the smaller the I, the faster the $\omega$ and vice versa.

For training of the jumping side volley, one should pay attention to the following three aspects: kick power, accuracy, and timing.

It is well known for practitioners that it is important to develop full-body whip-like control (from trunk to KS foot) for increasing kick power. For reaching this goal, jumping training should emphasize the upper-body twisting control, and the flexibility training should focus on the hip (one should always remember that the larger the $\beta$ is, the more powerful is the kick). The airborne training should establish an asymmetrical control of legs as well as the ROM training of the KS-leg joints, i.e., KS leg performing a whip-like control and NKS leg remaining extended.

Repetitive training is a traditional way to increase the aspects of kick accuracy and timing. Those of us who are involved in the coaching practice know well that there are no short-cuts. The jumping side volley is considered high risk because of the inevitable fall to the ground, i.e., a realistic fear of injury to players/learners. We know that the feet and legs are naturally designed for absorbing landing impacts, but the arms and body are not. What does that mean? That means that bio-adaptation training for strengthening the arms and body against impact and safe-fall training to minimize injury risk during falling to the ground should first be considered in skill learning [32]. Hence, if we apply the repetitive-training approach in learning the jumping side volley, we should begin with phase 3 , i.e., the learning and training of a safe falling technique, e.g., the multiple-landing technique revealed 
by the current study. Mastering a safe landing technique is the foundation that can ensure the repetitive training for improving kick accuracy and timing as well as the kick power.

Every study has limitations. As application-oriented research, the current study has two potential weaknesses. The obvious one is the low subject number and level. Future studies, if researchers cannot gain data from professional-level players, could first train advanced players based on the results of this study. As such, more subjects could be "produced" for future studies. The other one is the ecological approach to motor learning. For initiating the study, we simplified the ball movement. In reality, due to dynamic changes in the playing environment, a vertical travelling ball is only one of many possibilities. Therefore, identifying the learner-environment relationship as the basis for learning design in sport intentionality plays an important role in training. It will help to capture perception and action as intertwined processes underpinning individual differences in movement behavior [33,34]. The application of a nonlinear pedagogy, particularly in open and complex skills such as a jumping side kick, could reinforce the authors' intentions to help coaches to develop soccer-skills with their players. It would be a practitioner-desired topic for future studies.

\section{Conclusions}

The current study indicates one possibility to entrain the jumping side volley in soccer. Based on the results, the element training should focus on increasing the flexibility of the hip, the efficiency of the whip-like movement of the proximal-to-distal acceleration of the kicking leg, and the damping mechanism during falling. For skill training, one should focus on timing, because accurate timing is vital for a successful kick. One traditional way for improving timing is through repetitive training, which means repetitive falls to the ground during learning/training. Without safe fall protection, the skill learning/training presents a high risk of injury. This underlines the need for athletes to learn a safe landing technique that minimizes the risk of injury during practice. Therefore, if one applies the traditional approach in practice, training should begin with phase 3: mastering a safe fall.

Author Contributions: G.S., X.Z., F.L., and Y.Y. conceived and designed the experiments; G.S. and X.Z. performed the experiments; G.S., X.Z., F.L., and Y.Y. analyzed and discussed the data; G.S. and X.Z. contributed /materials/analysis tools; F.L. and Y.Y. prepared figures; G.S. and X.Z. wrote the paper; all authors contributed to the revisions and proof reading of the article. All authors have read and agreed to the published version of the manuscript.

Funding: The research project was supported by the National Sciences and Engineering Research Council of Canada (NSERC), grant number: RGPIN-2014-03648.

Conflicts of Interest: The authors declare no conflict of interest. The founding sponsors had no role in the collection, analyses, or interpretation of data; in the writing of the manuscript, and in the decision to publish the results.

\section{References}

1. FIFA. Average Number of Goals Scored per Game at the FIFA World Cup from 1930 to 2018. 2020. Available online: https://www.statista.com/statistics/269031/goals-scored-per-game-at-the-fifa-world-cup-since-1930/ (accessed on 26 January 2020).

2. Hyballa, P. The art of flying. Success Soccer 2002, 5, 19-26.

3. Reilly, T.; Williams, M. Science and Soccer, 2nd ed.; Routledge: London, UK, 2003.

4. Shan, G. Biomechanical Know-how of Fascinating Soccer-kicking Skills-3D, Full-body Demystification of Maximal Instep Kick, Bicycle Kick \& Side Volley. In Proceedings of the 8th International Scientific Conference on Kinesiology, Zagreb, Opatija, Croatia, 10-14 May 2017.

5. Shan, G.; Zhang, X.; Wan, B.; Yu, D.; Wilde, B.; Visentin, P. Biomechanics of coaching maximal instep soccer kick for practitioners. Interdiscip. Sci. Rev. 2019, 44, 12-20. [CrossRef]

6. YouTube. All 108 UEFA EURO 2016 Goals: Watch Every One. 2016. Available online: https://www.youtube. com/watch?v=a4Qvh6VIoSs (accessed on 26 January 2020).

7. FIFA. FIFA Puskás Award. 2019. Available online: https://www.fifa.com/the-best-fifa-football-awards/ puskas-award/ (accessed on 16 June 2019). 
8. UEFA. Top Ten Goals of UEFA EURO 2016 Revealed. 2016. Available online: https://www.uefa.com/uefaeuro2020/news/0253-0d7e907eeaa5-b7d7554f6864-1000--top-ten-goals-of-uefa-euro-2016-revealed/ (accessed on 19 September 2018).

9. Shan, G.; Visentin, P.; Zhang, X.; Hao, W.; Yu, D. Bicycle kick in soccer: Is the virtuosity systematically entrainable? Sci. Bull. 2015, 60, 819-821. [CrossRef]

10. Smith, P.G.; Morrow, R.H.; Ross, D.A. Preliminary studies and pilot testing. In Field Trials of Health Interventions: A Toolbox, 3rd ed.; Oxford University Press: Oxford, UK, 2015.

11. VICON. The Most Precise MoCap Ecosystem. 2020. Available online: https:/www.vicon.com/applications/ engineering/ (accessed on 26 January 2020).

12. Smith, S.L. Application of high-speed videography in sports analysis. In Ultrahigh-and High-Speed Photography, Videography, and Photonics; International Society for Optics and Photonics: Bellingham, WA, USA, 1993.

13. Chang, S.T.; Evans, J.; Crowe, S.; Zhang, X.; Shan, G. An innovative approach for Real Time Determination of Power and Reaction Time in a Martial Arts Quasi-Training Environment Using 3D Motion Capture and EMG Measurements. Arch. Budo 2011, 7, 185-196.

14. Wan, B.; Gao, Y.; Wang, Y.; Zhang, X.; Li, H.; Shan, G. Hammer Throw: A Pilot Study for a Novel Digital-route for Diagnosing and Improving Its Throw Quality. Appl. Sci. 2020, 10, 1922. [CrossRef]

15. Li, S.; Zhang, Z.; Wan, B.; Wilde, B.; Shan, G. The relevance of body positioning and its training effect on badminton smash. J. Sports Sci. 2017, 35, 310-316. [CrossRef] [PubMed]

16. Liu, Y.; Kong, J.; Wang, X.; Shan, G. Biomechanical analysis of Yang's spear turning-stab technique in Chinese martial arts. Phys. Act. Rev. 2020, 8, 16-22.

17. Michalowski, T. Applications of MATLAB in Science and Engineering; BoD-Books on Demand: Norderstedt, Germany, 2011.

18. Yu, D.; Yu, Y.; Wilde, B.; Shan, G. Biomechanical characteristics of the axe kick in Tae Kwon-Do. Arch. Budo 2012, 8, 213-218. [CrossRef]

19. Visentin, P.; Staples, T.; Wasiak, E.B.; Shan, G. A pilot study on the efficacy of line-of-sight gestural compensation while conducting music. Percept. Mot. Skills 2010, 110, 647-653. [CrossRef] [PubMed]

20. Shan, G.; Westerhoff, P. Full-body kinematic characteristics of the maximal instep soccer kick by male soccer players and parameters related to kick quality. Sports Biomech. 2005, 4, 59-72. [CrossRef] [PubMed]

21. Shan, G. Influences of Gender and Experience on the Maximal Instep Soccer Kick. Eur. J. Sport Sci. 2009, 9, 107-114. [CrossRef]

22. Shan, G.; Zhang, X. From 2D Leg Kinematics to 3D Full-body Biomechanics-The Past, Present and Future of Scientific Analysis of Maximal Instep Kick in Soccer. Sports Med. Arthrosc. Rehabil. Ther. Technol. 2011, 3 , 23. [CrossRef] [PubMed]

23. Shan, G.; Yuan, J.; Hao, W.; Gu, M.; Zhang, X. Regression equations for estimating the quality of maximal instep kick by males and females in soccer. Kinesiology 2012, 44, 139-147.

24. Shan, G.; Daniels, D.; Wang, C.; Wutzke, C.; Lemire, G. Biomechanical analysis of maximal instep kick by female soccer players. J. Hum. Mov. Stud. 2005, 49, 149-168.

25. Wan, B.; Shan, G. Biomechanical modeling as a practical tool for predicting injury risk related to repetitive muscle lengthening during learning and training of human complex motor skills. SpringerPlus 2016, 5, 441. [CrossRef] [PubMed]

26. Shan, G.; Bohn, C. Anthropometrical data and coefficients of regression related to gender and race. Appl. Ergon. 2003, 34, 327-337. [CrossRef]

27. Winter, D.A. Biomechanics and Motor Control of Human Movement; John Wiley \& Sons: Hoboken, NJ, USA, 2009.

28. Kearney, J.K.; Bhat, D.N.; Prasad, B.; Yuan, S.S. Efficient generation of whip-like throwing and striking motions. In Models and Techniques in Computer Animation; Magnenat-Thalmann, N., Thalmann, D., Eds.; Springer: Berlin, Germany, 1993; pp. 270-284.

29. Zhang, X.; Shan, G. Where do golf driver swings go wrong?-Factors Influencing Driver Swing Consistency. Scand. J. Med. Sci. Sports 2014, 24, 749-757. [CrossRef] [PubMed]

30. Zhang, Z.; Li, S.; Wan, B.; Visentin, P.; Jiang, Q.; Dyck, M.; Li, H.; Shan, G. The influence of X-factor (trunk rotation) and experience on the quality of the badminton forehand smash. J. Hum. Kinet. 2016, 53, 9-22. [CrossRef] [PubMed] 
31. Wąsik, J.A.; Ortenburger, D.O.; Góra, T.O.; Shan, G.O.; Mosler, D.; Wodarski, P.; Michnik, R.A. The influence of gender, dominant lower limb and type of target on the velocity of taekwon-do front kick. Acta Bioeng. Biomech. 2018, 20, 133-138. [PubMed]

32. Ballreich, R.; Baumann, W. Grundlagen der Biomechanik des Sports (The Basics of Biomechanics in Sports); Enke Verlag: Stuttgart, Germany, 1996.

33. Davids, K.; Araújo, D.; Hristovski, R.; Passos, P.; Chow, J.Y. Ecological dynamics and motor learning design in sport. In Skill Acquisition in Sport: Research, Theory and Practice, 2nd ed.; Hodges, N., Williams, M., Eds.; Routledge: London, UK, 2012; pp. 112-130.

34. Santos, S.D.; Memmert, D.; Sampaio, J.; Leite, N. The spawns of creative behavior in team sports: A creativity developmental framework. Front. Psychol. 2016, 7, 1282. [CrossRef] [PubMed]

(C) 2020 by the authors. Licensee MDPI, Basel, Switzerland. This article is an open access article distributed under the terms and conditions of the Creative Commons Attribution (CC BY) license (http://creativecommons.org/licenses/by/4.0/). 